\title{
Comunicación
}

\section{Frecuencia Histopatológica de Neoplasias Perianales en Caninos: Casuística del Laboratorio de Patología Veterinaria de la Universidad Nacional Mayor de San Marcos (2005-2012)}

\section{Histopathological Frequency of Perianal Neoplasms in Dogs: Casuistry of the Veterinary Pathology Laboratory of the National University of San Marcos} (2005-2012)

\author{
Karla Vicente R. ${ }^{1}$, Rosa Perales C., ${ }^{1,2}$ Luis Tabacchi N. ${ }^{1}$
}

\section{Resumen}

El objetivo del presente estudio fue determinar la frecuencia de neoplasias perianales en caninos mediante diagnóstico histopatológico en el Laboratorio de Patología Animal de la Universidad Nacional Mayor de San Marcos, Lima, Perú, en el periodo 2005-2012. Se diagnosticaron 52 casos de neoplasias perianales dentro de 1283 casos de neoplasias caninas (4.1\%). El adenoma de glándulas hepatoides fue el de mayor frecuencia $(46.2 \%$, 24/52).

Palabras clave: histopatología, frecuencia, neoplasia perianal, caninos

\section{Abstract}

The aim of this study was to determine the frequency of perianal neoplasms in dogs from samples histopathologically diagnosed in the Animal Pathology Laboratory of the National University of San Marcos in the period 2005 to 2012. A total of 52 cases of perianal tumors were diagnosed in 1283 cases of canine tumors $(4.1 \%)$. The hepatoid gland adenoma was the most frequent $(46.2 \%, 24 / 52)$.

Key words: histopathology, frequency, perianal neoplasm, canine

${ }^{1}$ Laboratorio de Histología, Embriología y Patología Animal, Facultad de Medicina Veterinaria, Universidad Nacional Mayor de San Marcos, Lima, Perú

${ }^{2}$ E-mail: rperales_fmv@hotmail.com 
En la sociedad actual, con el aumento de las expectativas de vida de las mascotas, consecuencia de los avances en la nutrición, técnicas diagnósticas, protocolos terapéuticos y medidas preventivas, viene resultando en una mayor incidencia de casos de cáncer (Martínez de Merlo et al., 2011).

Las neoplasias son un tema habitual en investigaciones científicas. En el Perú existen diversos estudios que describen la frecuencia de neoplasias a través de los años (Takano, 1964; Rodríguez, 1978; Castro, 1985; Díaz, 1991; San Martín, 2005), encontrando que las neoplasias de las glándulas hepatoides (sin especificar localización), adenoma y adenocarcinoma presentan frecuencias de $4.5,3.0$ y $1.7 \%$, respectivamente.

De Vivero et al. (2013) realizaron un estudio retrospectivo de 11 años (1995-2006) sobre la frecuencia de neoplasias caninas diagnosticadas en el Laboratorio de Histología, Embriología y Patología Animal (LHEPA) de la Universidad Nacional Mayor de San Marcos (UNMSM), reportando una frecuencia de $3.1 \%(34 / 1109)$ de adenomas y de $1.4 \%$ de adenocarcinomas de glándula perianal, aunque sin especificar la localización de las neoplasias (perianal, base de la cola, escroto o entrepierna). Por otro lado, Turek y Withrow (2009) refieren que las neoplasias de glándulas hepatoides representan hasta el $85 \%$ de neoplasias en esta región corporal.

El presente estudio se orienta a determinar la frecuencia de neoplasias en la región perianal, a fin de facilitar información al médico veterinario de práctica privada sobre estas neoplasias, ya que la mayoría de tumores o lesiones son asociados al adenoma o adenocarcinoma de glándulas perianales, instaurando a veces el tratamiento errado a falta de un adecuado diagnóstico histopatológico.
Estudio retrospectivo desarrollado con base a informes histopatológicos de neoplasias caninas de la región perianal del LHEPA-UNMSM, Lima, Perú, cubriendo el periodo de enero de 2005 a diciembre de 2012.

Se consideraron las siguientes variables: raza (pura y cruzada), edad ( 0 a menos de 1 , 1 a menos de 5, 5 a menos de 9 y 9 años a más) (Mendoza et al., 2010), sexo y diagnóstico histopatológico. Se determinó la frecuencia de neoplasias de la región perianal con relación al total de neoplasias en caninos.

\section{Resultados}

Se encontraron registros de 1283 neoplasias en canes, dentro de los cuales 52 fueron diagnosticadas como neoplasias perianales caninas, resultando una frecuencia de $4.1 \%$. La distribución porcentual por variable se presenta en el Cuadro 1. Las razas puras que presentaron más casos fueron el Samoyedo, Siberiano, Cocker Spaniel y Bóxer.

Cuadro 1. Distribución porcentual de 52 neoplasias perianales en canes según edad, sexo y grupo racial

\begin{tabular}{llc}
\hline Variable & & $\begin{array}{c}\text { Frecuencia } \\
(\%)\end{array}$ \\
\hline Edad & $0-<1$ & 2.4 \\
& $1-<5$ & 2.4 \\
& $5-<9$ & 35.7 \\
& $9->9$ & 59.5 \\
Sexo & Macho & 84.1 \\
& Hembra & 15.9 \\
\multirow{2}{*}{ Raza $^{1}$} & Pura & 66.7 \\
& Cruzado & 33.3 \\
\hline${ }^{1} \mathrm{n}=42$ & &
\end{tabular}


Cuadro 2. Neoplasias de la región perianal en caninos según el diagnóstico del laboratorio de histopatología (2005-2012)

\begin{tabular}{lcc}
\hline Diagnóstico histopatológico & $\begin{array}{c}\text { Casos } \\
(\mathrm{n})\end{array}$ & $\begin{array}{c}\text { Frecuencia } \\
(\%)\end{array}$ \\
\hline Adenomas de glándulas hepatoides & 24 & 46.2 \\
Adenocarcinoma de glándulas hepatoides & 16 & 30.8 \\
Carcinoma escamoso bien diferenciado ulcerado cutáneo perianal & 1 & 1.9 \\
Carcinoma papilífero de glándula sudorípara & 1 & 1.9 \\
Plasmocitoma extramedular perianal & 3 & 5.8 \\
Fibropapiloma asociado a quistes intradérmicos de inclusión cutáneo & 1 & 1.9 \\
perianal & 1 & 1.9 \\
Hemangiosarcoma perianal con presencia de cristales & 1 & 1.9 \\
Melanoma maligno tipo fusiforme dérmico ulcerado con quiste de & & \\
inclusión intradérmico & 1 & 1.9 \\
Rabdomiosarcoma perianal & 1 & 1.9 \\
Fibrosarcoma angiomatoso perianal con proceso inflamatorio & & \\
fibrinosupurativo & 1 & 1.9 \\
Linfangioma con quiste sebáceos & 1 & 1.9 \\
\hline Lipoma & 52 & 100 \\
\hline Total & & \\
\hline
\end{tabular}

Las neoplasias encontradas en la región perianal, según el diagnóstico histopatológico, se muestran en el Cuadro 2. El adenoma de glándulas hepatoides (Fig.1) presentó una frecuencia de $46.2 \%$, seguido del adenocarcinoma de glándulas hepatoides (30.8\%; Fig. 2) $\mathrm{y}$, en menor grado, el plasmocitoma extramedular perianal (5.8\%, Fig. 3).

\section{Discusión}

El 46.2\% de neoplasias de la región perianal correspondieron al adenoma de la glándula perianal, frecuencia menor que la reportada por Turek y Withrow (2009), mientras que el $30.8 \%$ fue para el carcinoma de glándulas hepatoides o perianal, frecuencia bastante mayor del 1\% indicado por Jubb et al. (1990) y del 3-21\% señalado por Pisani et al. (2006). Esto podría deberse a cierto rechazo en los dueños para realizar la este- rilización de sus mascotas, sobre todo si son machos.

La mayor frecuencia de neoplasias perianales ocurrió en los animales de mayor edad, resultado esperado pues se sabe que la edad es un factor de riesgo para la presentación de neoplasias malignas, sea cual fuera su estirpe celular (Martínez de Merlo y Pérez, 2007). En el presente estudio, el $48 \%$ de las neoplasias fueron malignas.

La mayor frecuencia de neoplasias perianales en razas puras podría deberse a mayores niveles de consanguinidad, dado que los criadores tienden a seleccionar pocos machos como reproductores (Frank y Nicholas, 1998). Por otro lado, la mayor frecuencia de neoplasias en canes machos estaría relacionado con el alto número de casos (24/52) de adenomas de glándulas hepatoides, dado que dicha neoplasia tiene una dependencia androgénica (Turek y Withrow, 2009). 


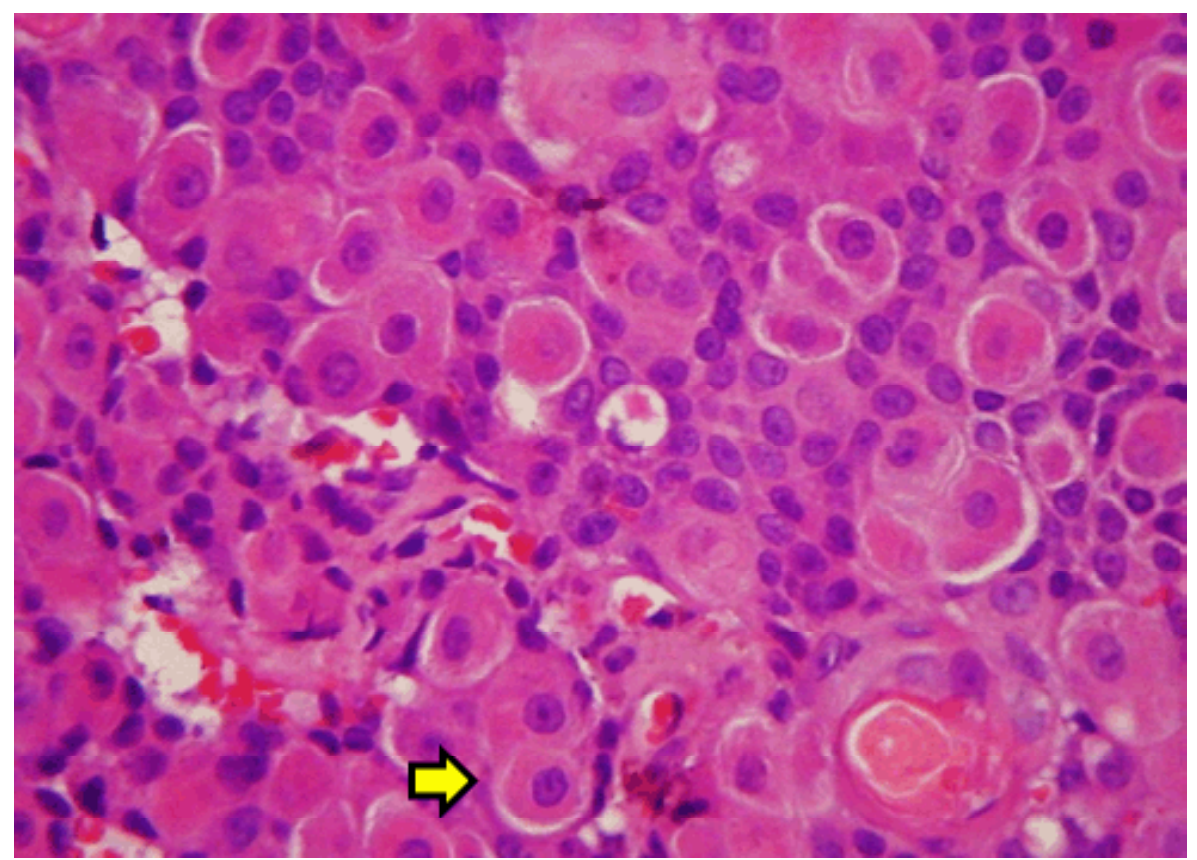

Figura 1. Adenoma de glándulas hepatoides en un perro macho, mestizo, de 7 años de edad. Se observan células homotípicas con núcleos bien delimitados y de posición central (flecha). $H \& E, 400 x$

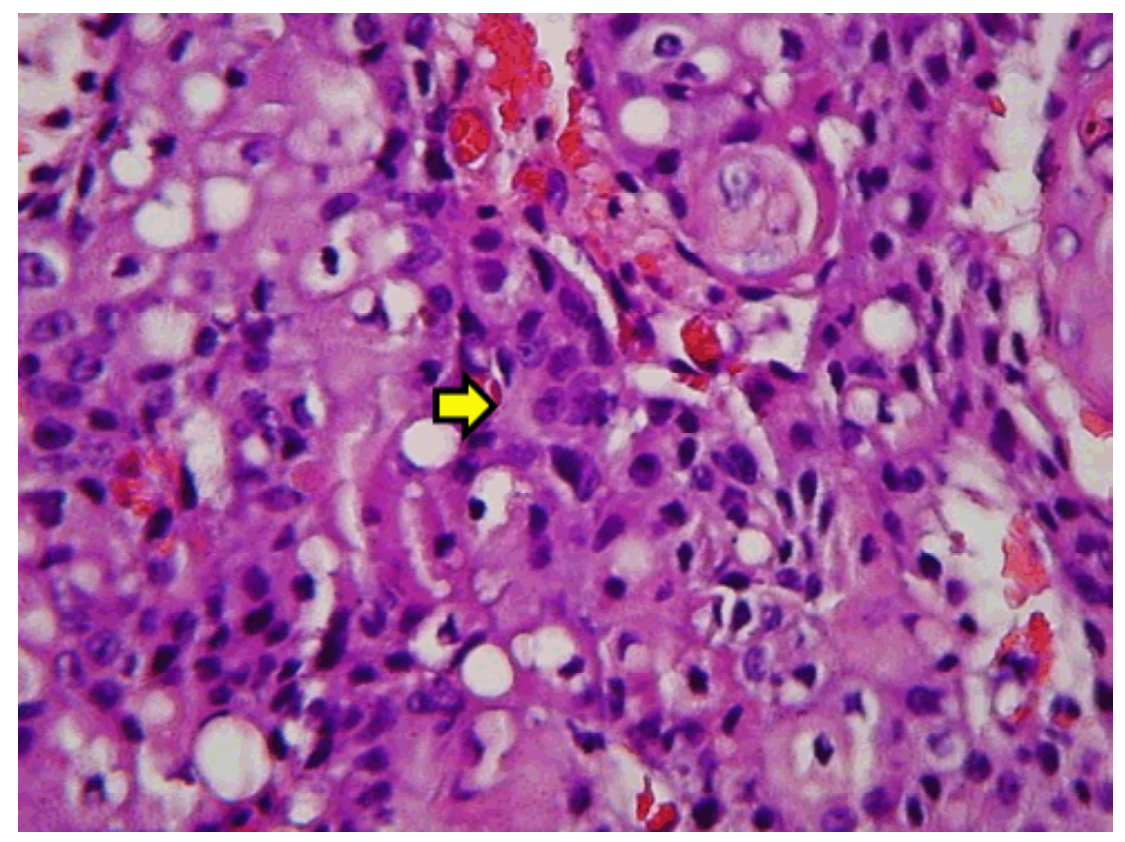

Figura 2. Adenocarcinoma de glándulas hepatoides en un Pastor Alemán, macho, de 12 años de edad. Se observan células de núcleos voluminosos, con anisocariosis, pleomorficas, binucleadas (flecha). H\&E, 400x 


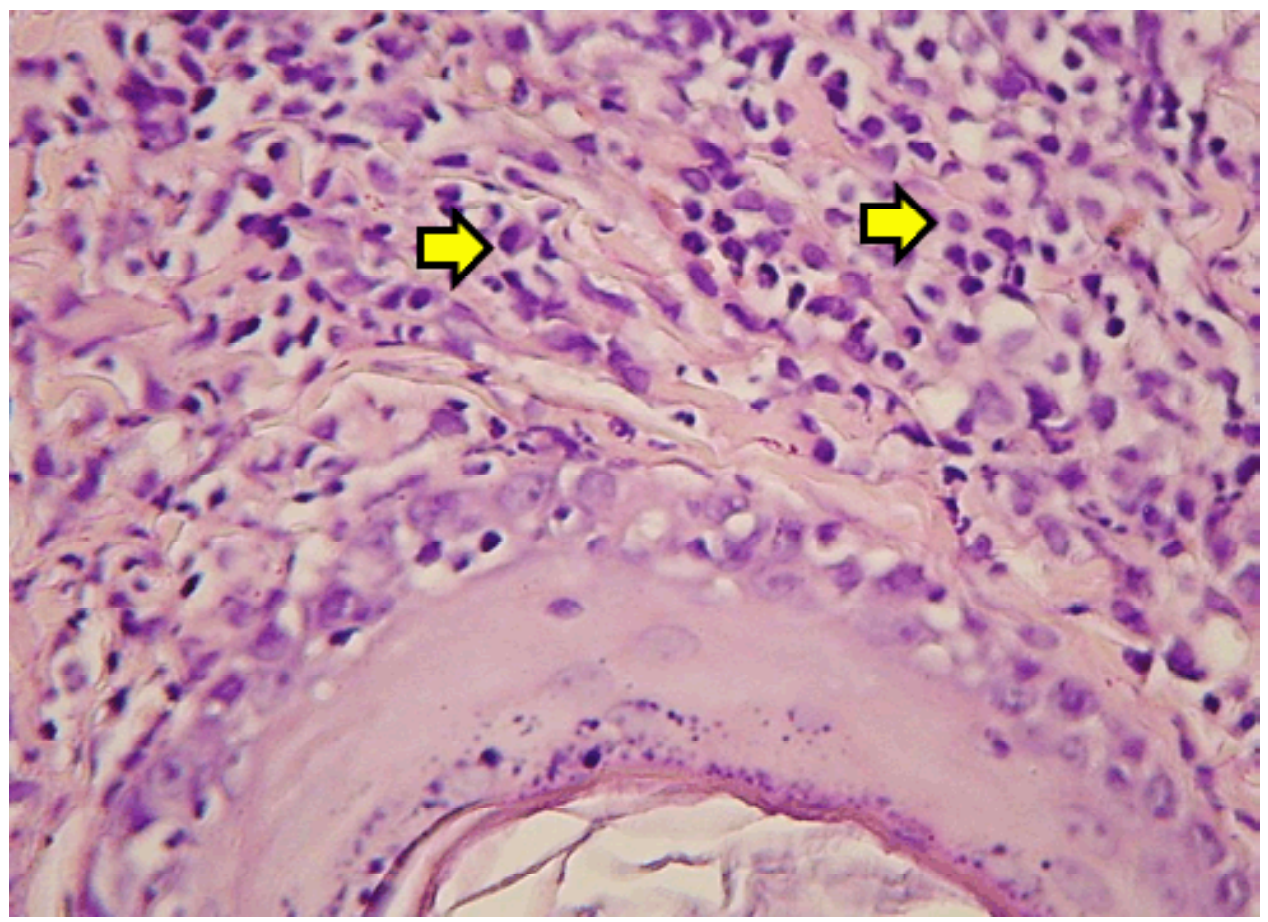

Figura 3. Plasmocitoma cutáneo perianal en un Cocker Spaniel hembra de 4 años de edad. Se observan células plasmáticas redondas con un núcleo hipercromático, localizado en posición central o excéntrica. H\&E, 1000x

\section{Literatura Citada}

1. Castro S. 1985. Frecuencia la presentación de neoplasias en caninos periodo 1978-1983. Tesis de Médico Veterinario. Lima: Univ Nacional Mayor de San Marcos. 46 p.

2. De Vivero L, Chavera A, Perales $R$, Fernández V. 2013. Frecuencia de neoplasias caninas en Lima: estudio retrospectivo en el periodo 1995-2006. Rev Inv Vet Perú 24: 182-188. doi: 10.15381/ rivep.v24i2.2487

3. Díaz V. 1990. Procesos neoplásicos en caninos. Análisis estadístico periodo 1984-1989. Tesis de Médico Veterinario. Lima: Univ Nacional Mayor de San Marcos. 34 p.

4. Frank WN. 1998. Introducción a la genética veterinaria. $2^{a}$ ed. España: Acribia. 362 p.
5. Jubb K, Kennedy J, Palmer N. 1990. Patología de los animales domésticos. Vol I. $3^{\text {a }}$ ed. Uruguay: Agropecuaria Hemisferio Sur. 671 p.

6. Martínez de Merlo M, Pérez C. 2007. Influencia de la edad en el desarrollo del cáncer. REDVET 2. [Internet]. Disponible en: http://www.veterinaria.org/revistas/recvet/n01a0407/01a040704.pdf

7. Martínez de Merlo E, Arenas C, Pérez D, Arconada L. 2011. Manual práctico de oncología en pequeños animales. España: Axón. 268 p.

8. Mendoza N, Chavera A, Falcón N, Perales $R$. 2010. Frecuencia del tumor venéreo transmisible en caninos. Casuística del Laboratorio de Patología Veterinaria de la Universidad Nacional Mayor de San Marcos (periodo 1998-2004). Rev Inv Vet Perú 21: 42-47. doi: 10.15381/ rivep.v21i1.305 
9. Pisani G, Millanta F, Lorenzi D, Vannozzi I, Poli A. 2006. Androgen receptor expression in normal, hyperplastic and neoplastic hepatoid glands in the dog. Res Vet Sci 81: 231-236. doi: 10.1016/ j.rvsc. 2005.11 .001

10. Rodríguez G. 1978. Neoplasias en la especie canina: estudio estadístico de 301 casos entre 1964-1977. Tesis de Médico Veterinario. Lima: Univ Nacional Mayor de San Marcos. 28 p.

11. San Martín M. 2005. Neoplasias caninas. Evaluación estadística. Perio- do 1990-1994. Tesis de Médico Veterinario. Lima: Univ Nacional Mayor de San Marcos. 46 p.

12. Takano F. 1964. Estudio retrospectivo de 154 procesos neoplásicos en la especie canina. Tesis de Médico Veterinario. Lima: Univ Nacional Mayor de San Marcos. $55 \mathrm{p}$.

13. Turek M, Withrow S. 2009. Tumores perianales. En: Withrow S, Vail D (eds). Oncología clínica de pequeños animales. $4^{\mathrm{a}}$ ed. España: Multimédica Ed Veterinarias. p 492-498. 